\title{
Homogenización computacional aplicada al estudio de suelos tipo conglomerado del austro ecuatoriano
}

\author{
Karla Santacruz, Esteban Samaniego \\ Facultad de Ingeniería, Universidad de Cuenca, Av. 12 de Abril s/n, Cuenca, Ecuador \\ E-mail:karlasantrey@hotmail.com, esteban.samaniego@gmail.com \\ Fecha de recepción: 28 de septiembre 2010 - Fecha de aceptación: 6 de noviembre 2010
}

\section{RESUMEN}

La ciudad de Cuenca, en el Austro Ecuatoriano, está asentada en su mayor parte sobre suelos tipo conglomerado (suelo heterogéneo constituido por una mezcla de materiales entre boleos decimétricos y arcilla). Actualmente, no existen valores de los parámetros mecánicos obtenidos experimentalmente, en gran medida debido a que los tamaños de los volúmenes representativos de este material son inmanejables en laboratorios. Ante esto, se utilizó una metodología basada en homogeneización computacional. El ingrediente central es un modelo computacional del material que permita realizar ensayos virtuales sobre volúmenes representativos. Para poder definir el modelo, debió determinarse las propiedades de cada elemento constitutivo del material. Se consideraron dos elementos: boleos, clastos con diámetros superiores a $3 / 4$ de pulgada, y matriz, material de dimensiones inferiores a $3 / 4$ de pulgada. Las propiedades de la matriz fueron halladas a través de ensayos triaxiales lentos saturados y triaxiales rápidos parcialmente saturados sobre muestras elaboradas con igual peso específico y humedad que el material de campo. Las propiedades del segundo elemento, los boleos, se determinaron a través de datos de la literatura al respecto. Para conocer el volumen a usar, se requieren una longitud característica y la distribución real de los elementos dentro del conjunto. El primer problema se resuelve mediante un análisis asintótico computacional. Para el segundo problema, se usaron fotografías digitales de taludes naturales o elaborados que muestren la distribución del conglomerado. Se realizó un análisis de sensibilidad de los factores más influyentes en las propiedades elásticas del conglomerado como compuesto (tamaño, forma, distribución y propiedades de los componentes). Usando el modelo computacional, las propiedades halladas para cada componente del conglomerado y variando los factores más influyentes, se llegó a obtener rangos de variación para el módulo de elasticidad (coeficiente de Young, E) y el coeficiente de Poisson $(v)$ del conglomerado en su conjunto.

Palabras clave: Homogeneización computacional, conglomerados, volumen representativo, boleos, matriz, propiedades elásticas.

\begin{abstract}
The soil type in Cuenca, Southern Ecuador, is called conglomerado (conglomerate, a heterogeneous soil composed of a mixture ranging from decimetric stones to clay). Experimental values of the characteristics of this material are hardly available, mainly because the size of the specimens is difficult manageable in a standard laboratory. To deal with this problem, the authors used a methodology based on computational homogenization. The main ingredient is a computational model of the material that allows performing virtual experiments over a representative volume. In order to build this model, the elastic properties of the components are experimentally defined. In the study two elements were considered: boulders, stones with diameters over $3 / 4$ of an inch, and matrix, the rest of the material. The matrix properties were found by performing triaxial tests on samples with the same specific weight and in situ moisture content. The properties of the other component, boulders, were derived from literature. The representative volume was determined on the basis of the characteristic length and the distribution of the components over the volume. The first issue was tackled by using a computational asymptotic analysis. The second issue was dealt with by using photographs of natural
\end{abstract}


slopes of the material. A sensitivity analysis to determine the most relevant factors (size, shape, distribution, components properties) was performed. Then, by using the properties of each component and allowing the relevant factors to vary, a range of values for the Young modulus (E) and the Poisson ratio $(v)$ of the composite were obtained.

Keywords: Computational homogenization, conglomerate, representative volume, matrix, elastic properties.

\section{INTRODUCCIÓN}

Las zonas de mayor desarrollo urbano de la ciudad de Cuenca se asientan sobre suelo de tipo conglomerado. Es decir, suelos de estructura granular con una gran variedad de tamaños. Este tipo de material es tratado de manera muy escasa en la literatura especializada que se enfoca más bien en los componentes finos y gruesos por separado. El rápido desarrollo urbano de la ciudad comienza a exigir soluciones arquitectónicas e ingenieriles más complejas, lo que significa que se debe hacer un mejor uso de su subsuelo. En este marco, no existe un modelo constitutivo adecuado para representar de manera idónea las relaciones entre esfuerzo y deformación a las cuales se verán sujetos los suelos en el caso de excavaciones a cielo abierto o en túnel, así como tampoco se conoce a profundidad la reacción de los suelos ante casos extremos de carga que pueden suponer estructuras más grandes o pesadas.

Para abordar este problema, se utiliza aquí una metodología basada en homogeneización computacional. El ingrediente fundamental es un modelo computacional de un volumen representativo de material para realizar ensayos virtuales. Con este modelo, se puede entonces obtener un rango de valores de los parámetros elásticos del material compuesto (el conglomerado) en función de diversos factores que pueden ser evaluados sin demasiada complejidad. Se busca, en particular, el poder determinar las propiedades elásticas del conglomerado a partir de las propiedades de la matriz (el componente fino, más fácilmente ensayable) y un conocimiento básico de otros factores (la fracción de volumen de sus componentes, por ejemplo).

La modelización constitutiva de geomateriales es especialmente complicada debido al hecho de que estos materiales no son homogéneos (Terzaghi y col., 1996) y, en general, no son producto de un diseño. Obtener un modelo constitutivo a partir de resultados puramente experimentales es una tarea complicada y costosa. En el caso específico de los conglomerados, la escala de un volumen estadísticamente representativo vuelve prohibitiva la tarea de determinación de parámetros geomecánicos mediante el uso exclusivo de ensayos experimentales. Tradicionalmente, el enfoque adoptado para la formulación de modelos constitutivos ha sido fenomenológico (Holzapfel, 2006). Esto es, se ha elaborado el modelo a base de mediciones experimentales que no tenían en cuenta el micro- o meso-estructura, según sea el caso, del material modelizado. Una segunda forma para llegar al modelo constitutivo de un material es usar la teoría de la homogeneización (Nemat-Nasser y Hori, 1999; Zohdi y Wriggers, 2004). Grosso modo, la homogeneización consiste, para empezar, en considerar una especie de promedio ponderado de las cantidades físicas que intervienen en la descripción del comportamiento de un material heterogéneo en una región representativa. Se hallan, entonces, relaciones entre estos promedios. Dichas relaciones caracterizarán al material considerado como un todo homogéneo.

Un concepto fundamental en la teoría de la homogeneización es el Elemento de Volumen Representativo (EVR). Un EVR es el mínimo volumen de material cuyo comportamiento es mecánicamente equivalente a un volumen de un material ficticio homogéneo. El estudio de un EVR de un material heterogéneo permite determinar las ecuaciones constitutivas de un material homogéneo mecánicamente equivalente. En el caso de los conglomerados, la definición del EVR en sentido estricto no es posible, debido a que la distribución de los componentes es aleatoria. El término EVR tal como se usa en este artículo corresponde más bien al sentido dado en Ostoja-Starzewski (2006) al término Elemento de Volumen Estadístico. Se prefiere en aquí el término EVR por simplicidad conceptual. En este trabajo, se usa la herramienta computacional desarrollada por los autores en Samaniego y col. (2008), además de algunos de los resultados obtenidos en esta referencia. Un 
componente importante de la metodología desarrollada es la obtención de la información de la conformación geométrica del volumen representativo a partir de fotografías de taludes. Antecedentes de este tipo de enfoque se pueden encontrar por ejemplo en Kanit y col. (2003) y Terada y col. (2002).
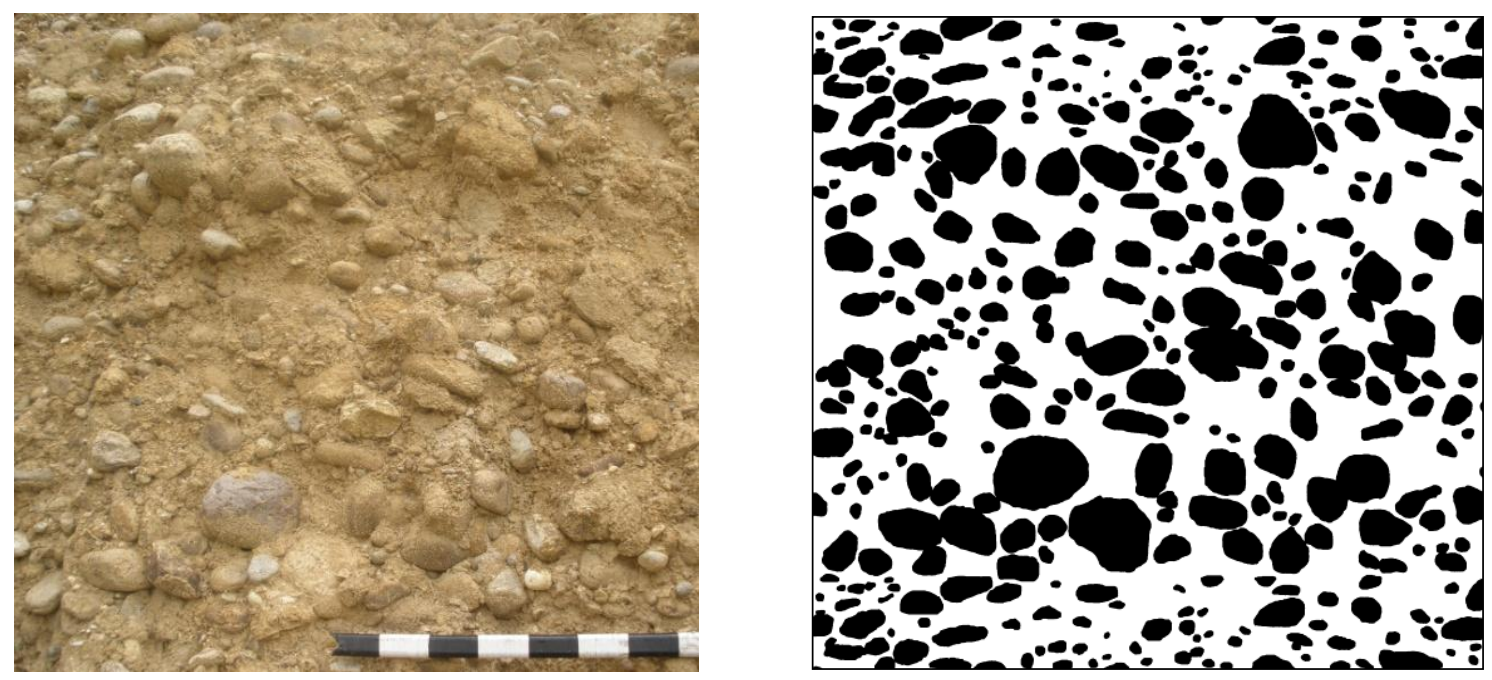

Figura 1. Proceso de segmentación de imágenes. A la izquierda la fotografía original; a la derecha la imagen segmentada.

\section{MATERIALES Y MÉTODOS}

El presente trabajo estudia conglomerados de la ciudad de Cuenca. Debido a la gran cantidad y diferencias entre los conglomerados hallados en la ciudad, se restringe el análisis a conglomerados pertenecientes a dos formaciones geológicas Terrazas Fluvioglaciares y Formación Turi. Inclusive dentro de las Terrazas Fluvioglaciaricas debió limitarse el análisis a dos tipos de terrazas, las terrazas 3 y 4.

Las Terrazas Fluvioglaciaricas, conocidas también como Terrazas Aluviales, son depósitos recientes del Cuaternario. Se las considera formadas por material producto del fenómeno glaciar acarreado por grandes corrientes fluviales (Samaniego y col., 2008). Actualmente las terrazas se ubican principalmente alrededor de los ríos de la ciudad y están constituidas en su mayor parte por conglomerados de matriz areno arcillosa. Según la composición, edad y ubicación, las terrazas se dividen en cinco grupos Terraza 1 a la 5. Luego de una caracterización física de las terrazas de cada nivel, es decir de un estudio de sus propiedades físicas tales como granulometría y límites de Atterberg, se llego a determinar cierta semejanza entre las terrazas 3 y 4 y a su vez de estas con el conglomerado de la Formación Turi. Es por esto que se eligen las Terrazas 3, 4 y la Formación Turi como materiales dentro del presente estudio.

Por otro lado la Formación Turi es una de las formaciones más jóvenes dentro de la geología de la ciudad de Cuenca. Se la considera un depósito continental de origen fluvial y con aportes volcánicos (Erazo, 2009; Samaniego y col., 2008; Nuñez y Orellana, 2009). Está compuesta de conglomerados, arenas, arcillas, tobas y brechas. Por su puesto que el material de la formación analizado en el presente trabajo es únicamente el conglomerado.

Como se mencionó anteriormente, se realizó una caracterización previa de los conglomerados de cada formación. Los resultados obtenidos indican dos principales clasificaciones según el Sistema Unificado de Clasificación de los Suelos (SUCS): grava mal graduada y grava bien graduada. Los porcentajes de clastos varían aproximadamente entre 25 y $57 \%$. Materiales con esta clasificación son los analizados mediante los métodos que se resumen a continuación. 
El procedimiento para determinar los parámetros que definen el comportamiento de ciertos conglomerados en el rango elástico usado en este trabajo es el meta de los autores Samaniego y col. (2008). Este procedimiento combina ensayos experimentales y simulaciones numéricas. Los ensayos se hacen, en principio, sobre la matriz y sobre los boleos por separado. Las simulaciones (ensayos virtuales) se realizan sobre volúmenes de conglomerado a fin de obtener las propiedades elásticas del conjunto.

Para hacer viable la modelación de los conglomerados en estudio, se divide el conglomerado en dos componentes: matriz, material con tamaño inferior a $3 / 4$ de pulgada y boleos, y el material restante (tamaños mayores). Se procede entonces a obtener las propiedades elásticas de cada componente. Para la matriz se realizaron ensayos triaxiales consolidados drenados y no consolidados no drenados sobre muestras elaboradas en laboratorio, conservando el peso específico y la humedad natural del material en campo. Se eligió el uso de los dos tipos de triaxiales con la finalidad de ampliar los resultados para los dos tipos de comportamientos extremos que el material podría tener. Además, se varió la presión de cámara que correspondería a las diferentes presiones de confinamiento a las que el material pueda estar sometido. El peso específico natural de la matriz se obtiene a través de ensayos en campo. Se realizaron ensayos tipo globo de hule, pero llevados a cabo de una manera artesanal buscando abordar la mayor cantidad de volumen posible. Como los resultados se obtienen dentro de un rango de variación, los ensayos triaxiales debieron efectuarse dentro de ese rango y no exclusivamente para un solo dato de peso especifico. Para los boleos se eligieron propiedades de bibliografía especializada, en función del tipo de roca dominante en los conglomerados estudiados.

Por otra parte y con el objeto de definir los modelos computacionales de los diferentes volúmenes de conglomerado, se necesita la geometría de dicho volumen, esto es, la distribución geométrica de los boleos en la matriz. Para esto se parte de fotografías de taludes del conglomerado que se quiere estudiar. Estas fotografías se someten a un proceso de segmentación de imágenes, como se muestra en la Figura 1, para separar las regiones en las que se encuentran los boleos de aquellas que corresponden a la matriz. Una vez hecho esto, se procede a vectorizar la imagen, es decir, pasar de una descripción de la imagen basada en píxeles a una descripción de tipo CAD. Esto último se hace a fin de que la imagen segmentada pueda ser leída por un programa de generación de mallas de elementos finitos. Una vez generada la malla, esta se introduce en un programa de elementos finitos. En este momento se usa la información de las propiedades de la matriz y los boleos obtenida en laboratorio. Con la malla generada y las propiedades materiales, se requiere imponer unas condiciones de contorno para completar la definición del modelo de elementos finitos de un volumen de conglomerado. Estas condiciones de contorno se obtienen usando teoría de homogeneización y corresponden a ensayos (virtuales) convenientemente elegidos para determinar las propiedades del conjunto. En este caso se usaron uno de deformación volumétrica y otro de cortante puro.

A fin de realizar el proceso de homogeneización computacional y encontrar los parámetros elásticos del conjunto, es imprescindible contar con el tamaño de un Elemento de Volumen Representativo de conglomerado. Para esto, se adopta en este trabajo el máximo de los valores obtenidos en Samaniego y col. (2008). Estos valores se obtuvieron repitiendo el proceso descrito en los párrafos anteriores para volúmenes de diversos tamaños. De esta manera se obtienen parámetros aparentes para diversos tamaños de volúmenes. La observación de su comportamiento asintótico permite, entonces, inferir valores efectivos para las propiedades. Se entiende aquí por valores efectivos a aquellos que son estadísticamente representativos del comportamiento del conglomerado. Este proceso asintótico se debe realizar debido a que los conglomerados son materiales no periódicos $\mathrm{y}$, por tanto, un volumen representativo en sentido estricto debería tener tamaño infinito.

Contando con la información necesaria - propiedades de los componentes del conglomerado, geometría y distribución de boleos en conglomerados reales y tamaños representativos de las muestras a usar - se procedió a un estudio de sensibilidad de las diferentes variables en las propiedades elásticas del material en conjunto. Se analizaron factores como el porcentaje de boleos presentes en las muestras, la gradación de las partículas de boleos (su granulometría), el tamaño y la forma de los boleos y por último su distribución dentro de la matriz. Se ejecutaron un sinnúmero de ensayos virtuales variando cada parámetro, dentro de rangos posibles. A través de estos ensayos, se determina la influencia de cada factor en las propiedades elásticas del conglomerado. Se eligen las propiedades que provocan notoria influencia y en función de ellas se llega a determinar los rangos posibles de 
variación de las propiedades elásticas (módulo de elasticidad y coeficiente de Poisson) para los conglomerados estudiados. Detalles de estos ensayos se pueden encontrar en Santacruz (2009).

Usando el modelo establecido, las propiedades halladas para cada componente del conglomerado (sus rangos) y variando los factores más influyentes en las mismas, se llega a obtener rangos de variación para el módulo de elasticidad y el coeficiente de Poisson del conglomerado. Es así como este trabajo permite llegar a pronosticar valores para las propiedades elásticas del conglomerado (ecuaciones en función del porcentaje de boleos y módulo de elasticidad de la matriz) partiendo de un conocimiento básico del mismo: su granulometría, porcentaje de boleos, profundidad a la que se requiere el análisis y comportamiento elástico de los componentes, en especial el comportamiento elástico de la matriz.
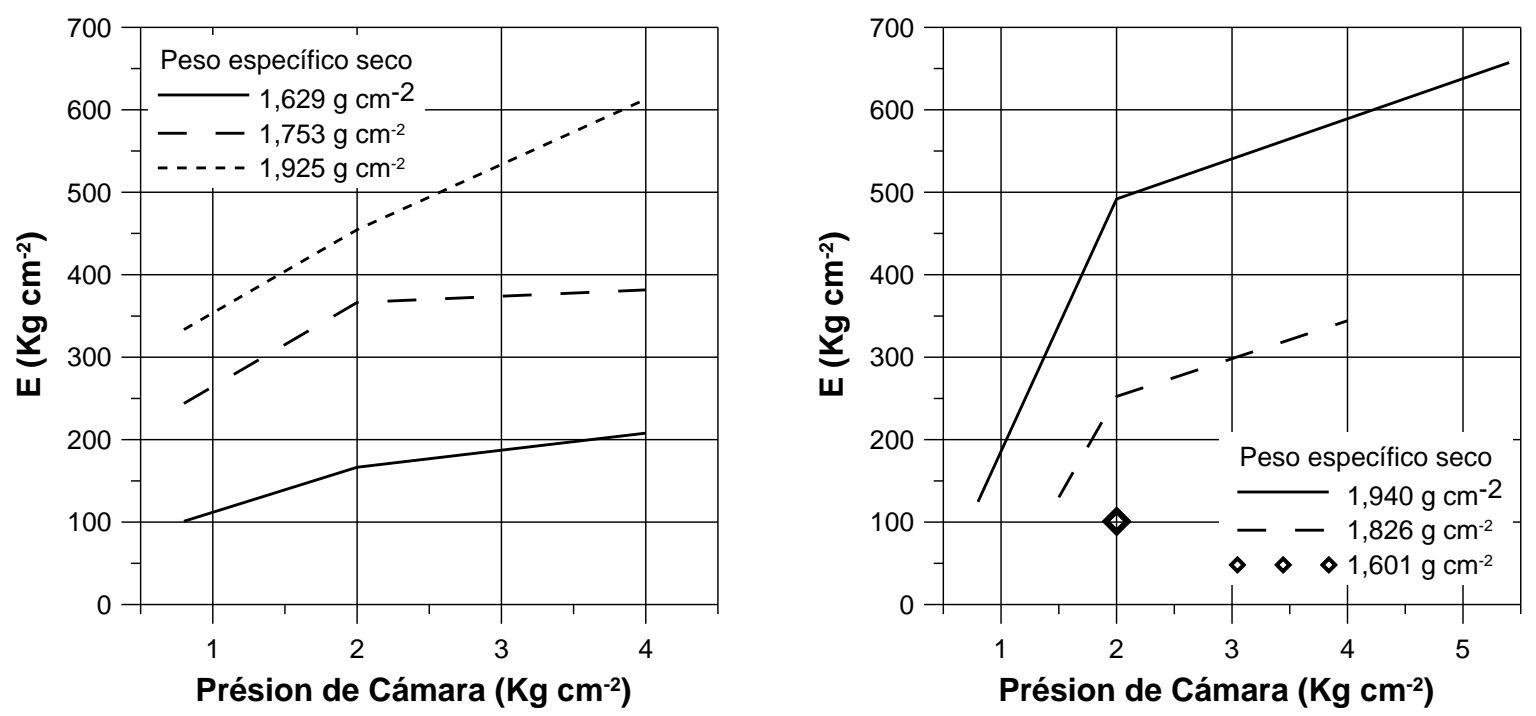

Figura 2. Propiedades elásticas de la matriz. A la izquierda: resultados triaxiales rápidos; a la derecha: resultados triaxiales lentos.

\section{RESULTADOS}

La determinación de las propiedades elásticas de la matriz trae consigo algunas variables de influencia. En primer lugar, los pesos específicos hallados en campo presentan un rango de variación entre 1,6 y $2,0 \mathrm{~g} \mathrm{~cm}^{-3}$. Por lo tanto, los ensayos triaxiales sobre las muestras de matriz debían ejecutarse con diferentes pesos específicos dentro del rango mencionado. Otra variable a considerar resulta la presión de confinamiento a ser usada dentro de cada ensayo triaxial, esto está en función de la profundidad de análisis de las muestras. Por lo tanto, se ensayan muestras con diferentes presiones de confinamiento, entre 0,5 y $5,0 \mathrm{Kg} \mathrm{cm}^{-2}$, considerando profundidades hasta los $40 \mathrm{~m}$ como factibles de aplicación. Los resultados obtenidos en cuanto a propiedades elásticas se resumen en la Figura 2. Dentro de los estudios de los boleos, según los análisis en campo, se puede llegar a determinar una importante presencia de roca andesita dentro de los conglomerados analizados; además existen bajos porcentajes riolita, dioria e inclusive basalto. En función de la presencia de materiales, se determinaron las propiedades más adecuadas a usar. Para el módulo de elasticidad de los boleos se elige el valor de $300.000 \mathrm{Kg} \mathrm{cm}^{-2}$, aunque se realiza además un chequeo de sensibilidad en los resultados finales considerando variaciones de este parámetro. Para el coeficiente de Poisson, se usa un valor de 0,28.

En cuanto al método computacional usado para el ensayo virtual de las muestras de conglomerado se utilizan fotografías de conglomerados reales de la ciudad de Cuenca; su tamaño mínimo debe ser de 1,4 m x 1,4 m que resulta el tamaño máximo desde donde los resultados de las 
propiedades del conglomerado empiezan a comportarse como una asíntota. Partiendo de la fotografía se usó un programa que segmenta la fotografía transformándola en dos elementos: boleos y matriz, cuidando los tamaños elegidos como límite. Se usaron técnicas basadas en umbralización, crecimiento de regiones, y detección de contornos. Esta técnica depende del tipo de imagen con la que se trabaje. En cuanto a la elección del método más adecuado a usar, se puede consultar Nuñez y Orellana (2009). Hay conglomerados que muestran un cambio de color entre matriz y boleos resultando muy fácil la separación de componentes; otros en los que los boleos difícilmente se pueden diferenciar de la matriz incluso al ojo humano. Es complicado que de todas las fotografías se pueda obtener una segmentación aceptable. En casos complicados se debió proceder a un tipo de segmentación manual a través de un programa de manipulación de imágenes, como, por ejemplo GNU Image Manipulation Program for X Windows (GIMP) o Photoshop, de manera similar a la que se menciona en Terada y col. (2002). En esta segmentación manual deben pintarse todos los boleos con diámetro superior a 3/4 de pulgada. Mediante el uso de una rejilla se determina el tamaño mínimo requerido. Es un proceso laborioso pero con resultados tan buenos que resultan casi una copia de la fotografía original.

Para la mayoría de casos, se usa el programa computacional para segmentación desarrollado en Samaniego y col. (2008). Este requiere el ingreso de algunos datos de cada fotografía, estos son la escala pixel-metro, área mínima de boleo y la longitud característica, que es un factor que trabaja con el grado de separación entre boleos, ya que si se encuentran muy juntos el programa tiende a unirlos. Los valores mencionados varían su rango según el tipo de fotografía y, sobre todo, según la escala de la misma, así que debía ensayarse cada muestra con varios parámetros hasta obtener los más adecuados, es decir, los que generan los mejores resultados, la mayor similitud con la fotografía original. La fotografía segmentada se convierte de formato raster a formato vector. Con el resultado obtenido se ingresa al programa GMSH (Geuzaine y Remacle, 2008) que genera una malla a partir de la imagen segmentada ya en formato vector.

Una vez con los resultados de las propiedades elásticas de los componentes del conglomerado, se realizó un análisis de sensibilidad con el propósito de determinar los factores más influyentes en la variación de las propiedades elásticas del conglomerado en conjunto. El factor más obvio que generaría variación en las propiedades elásticas del conglomerado resulta la cantidad de boleos presentes en la muestra, a mayor cantidad de boleos mayor será el módulo de elasticidad del conglomerado. Se determina la cantidad de boleos presentes en cada muestra fotográfica segmentada, para ello se usa la herramienta histograma de GIMP, que es capaz de determinar el porcentaje de pixeles en negro (boleos). Las muestras analizadas exhiben los porcentajes de boleos de la Tabla 1.

Tabla 1. Módulo de elasticidad (E) y coeficiente de Poisson (v) para el conglomerado de siete muestras.

\begin{tabular}{lccc}
\hline \multicolumn{1}{c}{ Locación } & $\begin{array}{c}\text { Boleos } \\
(\%)\end{array}$ & $\begin{array}{c}\mathrm{E} \\
\left(\mathrm{Kg} \mathrm{cm}^{-2}\right)\end{array}$ & $\begin{array}{c}\text { Coeficiente de } \\
\text { Poisson }(v)\end{array}$ \\
\hline Terraza 3_esc187 & 40,09 & 252,17 & 0,211 \\
Terraza 3_esc130 & 28,84 & 188,56 & 0,215 \\
Terraza 3_esc158 & 43,73 & 297,51 & 0,213 \\
Terraza 4_esc170 & 30,98 & 199,80 & 0,212 \\
Terraza 4_esc170 & 32,99 & 209,78 & 0,213 \\
Terraza 4_esc135 & 51,98 & 415,97 & 0,252 \\
Formación Turi_esc106 & 31,45 & 199,10 & 0,212 \\
Formación Turi_esc230 & 24,82 & 155,80 & 0,212 \\
\hline
\end{tabular}

Los porcentajes en volumen de boleos encontrados según las muestras fotográficas analizadas varían entre 24 y 53\%. Comparando con los porcentajes de boleos obtenidos según análisis en muestras físicas en laboratorio de 25 y $57 \%$ (porcentajes en volumen). Los valores son comparables, es decir muestran un rango de variación muy similar. Debido a la importante variabilidad que 
presentan los conglomerados en cuanto a porcentaje de boleos dentro de su estructura, y a su influencia en los resultados de las propiedades de los conglomerados (según resultados de ensayos que se indican posteriormente), se considera importante usar siempre una relación entre el módulo de elasticidad del conglomerado y su porcentaje de boleos, es decir, de ahora en adelante todos los análisis se realizan en base a la o las curvas obtenidas de E vs. \% boleos. Para la relación de Poisson (la segunda propiedad elástica buscada) se usaría igual criterio.
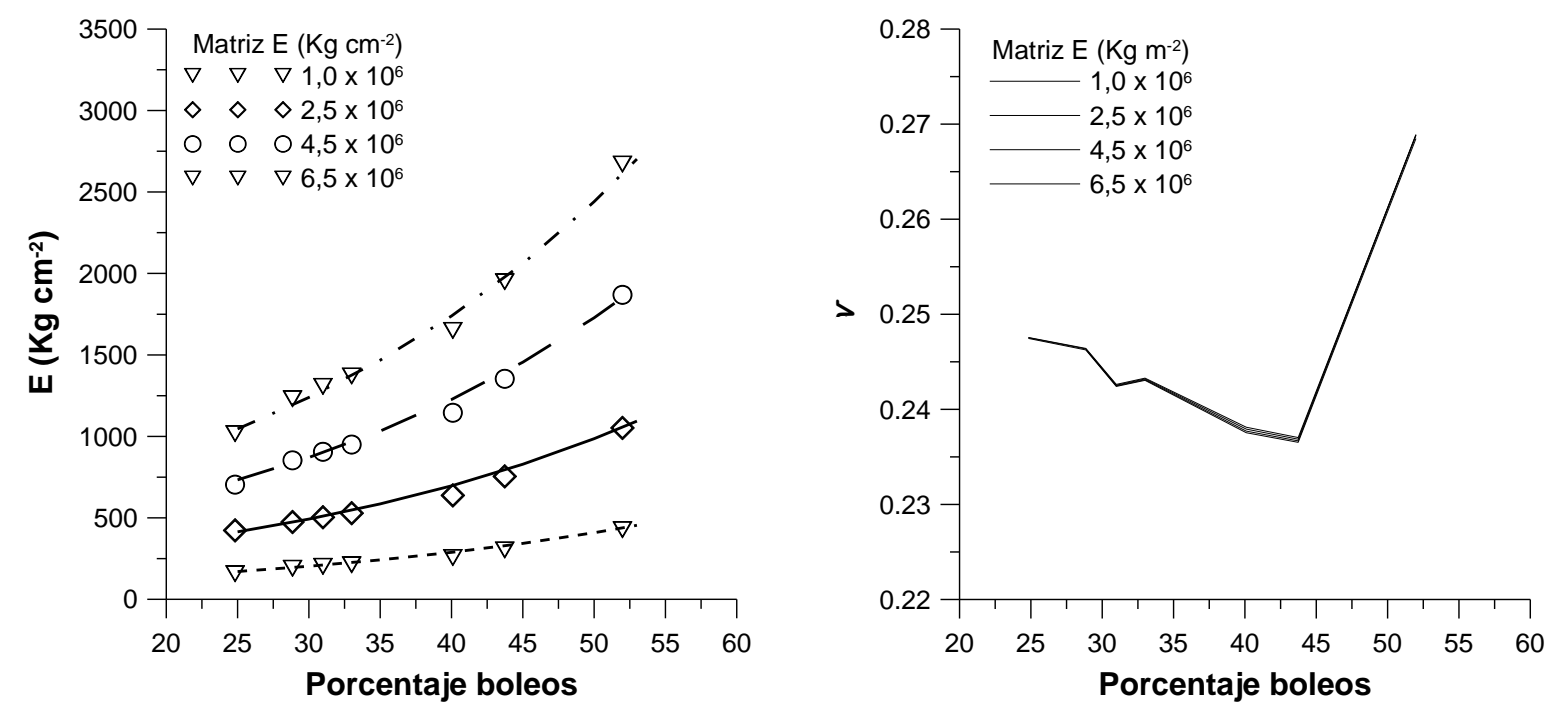

Figura 3. Propiedades elásticas del conglomerado obtenidas en función del porcentaje de boleos. A la izquierda: módulo de elasticidad (E) de conglomerado, resultados experimentación (símbolos) y ecuación (línea); a la derecha: coeficiente de Poisson (v).

Posteriormente se realizó un estudio de sensibilidad variando la distribución de los boleos en la muestra, su granulometría, las propiedades elásticas de los boleos y su tamaño. Los ensayos consistieron en variar, dentro de un rango posible únicamente cada parámetro y revisar su correspondiente influencia en las propiedades del conglomerado como conjunto. Se llegó a determinar que si se trabaja únicamente dentro de las granulometrías normales para los conglomerados estudiados, las propiedades del conjunto no son afectadas por las variaciones de los parámetros mencionados. Entonces se considera como único parámetro de variación la cantidad de boleos presentes en las muestras además claro de las propiedades de la matriz. Los resultados de las propiedades de los conglomerados se obtienen en función a la variación de los parámetros influyentes en su valor. Se debe por lo tanto partir con propiedades de la matriz, que a su vez deben elegirse en función de sus correspondientes parámetros de variación, y de la cantidad de boleos presentes en el conglomerado en estudio. Los resultados obtenidos se indican en la Figura 3.

\section{DISCUSIÓN}

Los resultados obtenidos permiten contar con relaciones entre las propiedades elásticas del conglomerado y propiedades relativamente fáciles de determinar. El módulo de Young (E) de la matriz y el porcentaje de boleos del conglomerado resultan ser los factores determinantes en el comportamiento del conjunto. Esto implica claras ventajas prácticas. Sin embargo, está claro que, a pesar de las sólidas bases mecánicas que fundamentan la teoría de la homogeneización, lo cual hace plausible la confiabilidad de los resultados, una validación experimental de estos se presenta como indispensable. 
Aunque no se cuenta con una validación física de los resultados obtenidos, se los puede comparar con información sobre módulos de elasticidad obtenidos de ensayos reales sobre materiales con características granulométricas muy similares a las halladas para el conglomerado. Alemán Velásquez y col. (2009) se presenta un estudio acerca de la construcción y seguimiento al comportamiento geotécnico de una presa de enrocado del proyecto hidroeléctrico La Yesca, localizada sobre el río Santiago cerca de la ciudad de Guadalajara, México. En su desarrollo la publicación indica la obtención de módulos de elasticidad reales conseguidos mediante ensayos triaxiales gigantes sobre materiales con granulometrías específicas.

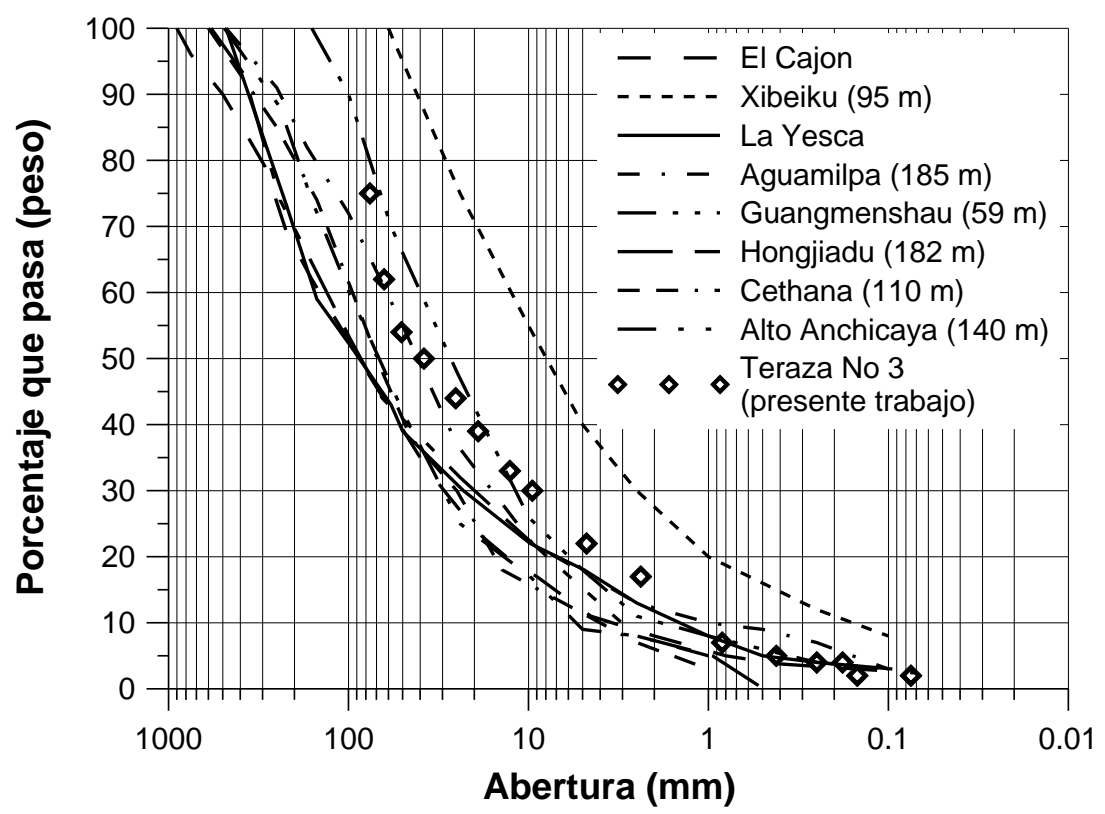

Figura 4. Curvas granulométricas para diferentes tipos de materiales empleados en la construcción de presas de tierra en México. Grafico obtenido de Alemán Velásquez y col. (2009). Se incluyen puntos aislados que representan la granulometría hallada para una Terraza No 3 (estudiada en el presente trabajo).

Se indica en la Figura 4 un gráfico obtenido de esta publicación en donde se pueden observar las diferentes curvas granulométricas típicas para cada material del cual posteriormente se obtienen sus módulos de elasticidad, comparada con resultados obtenidos en el presente trabajo. En la Figura 5, tomada de la misma referencia, se muestran precisamente los módulos de elasticidad obtenidos para cada material en función de la presión de confinamiento usada. La Figura 4 muestra las curvas granulométricas para los materiales estudiados. Se incorpora en el gráfico una granulometría de conglomerado de la Terraza 3 estudiada en el presente trabajo. Esta granulometría se ubica entre las curvas mostradas en la figura (corresponde a los puntos que no están unidos por una línea), lo que quiere decir que la composición de un conglomerado de Terraza 3 es comparable con los materiales que estudia la referencia mencionada.

La Figura 5 indica la variación del módulo de elasticidad de los materiales estudiados en Alemán Velásquez y col. (2009) en función de la presión de confinamiento. Se incorporó una escala en $\mathrm{Kg} \mathrm{cm}^{-}$ ${ }^{2}$ que es la unidad de presión usada en el presente trabajo. La elipse indica el rango en donde se ubican los resultados de módulo de elasticidad para el conglomerado estudiado en el presente trabajo. Se puede observar cómo los resultados para el conglomerado se ubican dentro del rango de valores reales resultado de ensayos físicos sobre materiales de similares características. Cabe recalcar que los materiales que sirven de referencia son materiales elaborados, no naturales; es decir son comparables a los efectivamente usados en el presente trabajo. La diferencia radica en que los primeros resultados se obtienen de ensayos físicos sobre las muestras mientras en los segundos (los correspondientes a este artículo) resultan de experimentación numérica en computador. Según esta comparación, los 
módulos de elasticidad del conglomerado tienen un cierto grado de confianza al resultar semejantes a las propiedades obtenidas a través de ensayos físicos (triaxiales) sobre muestras de composición similar. Queda, claro está, la pregunta de cuánto se alteran las muestras al reconformarlas para su estudio en laboratorio.

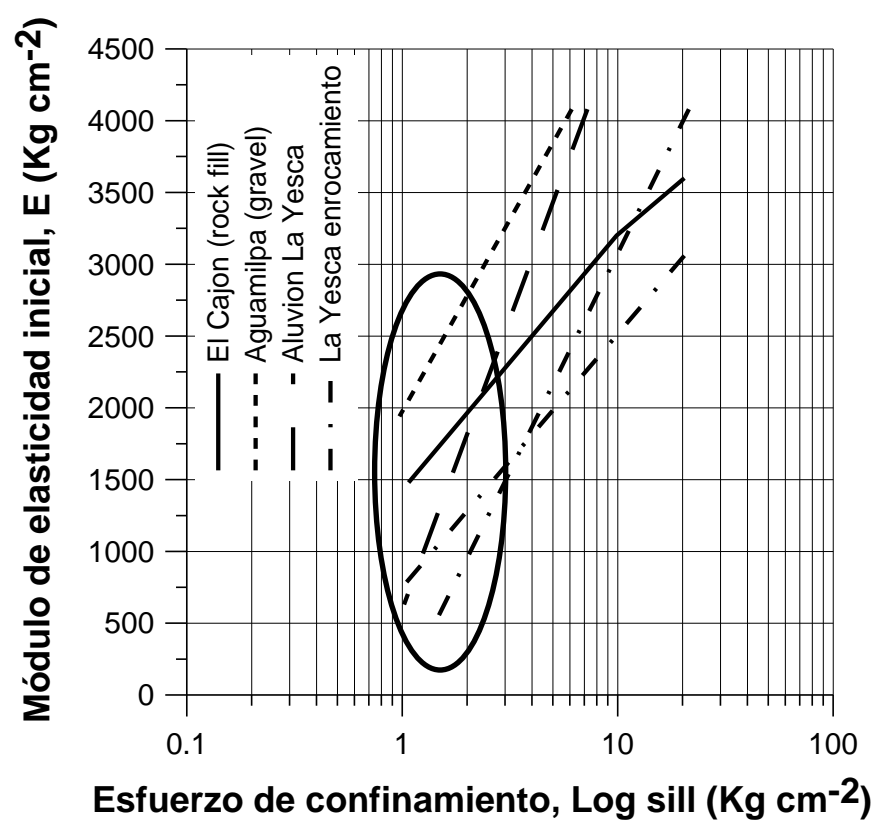

Figura 5. Resultados de módulos de elasticidad de los materiales indicados en la Figura 4 en función del esfuerzo de confinamiento. Obtenido de Alemán Velásquez y col. (2009). La elipse representa la zona donde se encuentran los resultados del presente trabajo.

\section{CONCLUSIONES}

El presente trabajo ha llegado a determinar una relación entre las propiedades elásticas del conglomerado en función de las propiedades de su matriz y del porcentaje de boleos presentes en el conjunto (conglomerado). Para estimar un valor de módulo de Young y de Poisson para un conglomerado específico, se debería proceder de la siguiente manera. En primer lugar es necesario contar con cierta información del conglomerado en conjunto. El porcentaje de boleos presentes es uno de los parámetros más importantes. Se lo puede aproximar a través de un análisis visual o, de ser posible, de una granulometría en un volumen representativo. Por otro lado, se necesitaría saber si el conglomerado tiene características asimilables a las de un conglomerado estándar estudiado en el presente trabajo. Una buena manera de determinarlo es comparando la granulometría del conglomerado que se quiere estudiar con la presentada aquí. Por último, se debería contar con información sobre la matriz del conglomerado. Este aspecto es de más cuidado y requiere mayor análisis. Se debería llegar a determinar el peso especifico in situ de la matriz, además de establecer la profundidad de estudio requerida y el ensayo triaxial que más se asemeja al posible comportamiento del material en campo. Con esta información, usando la figura 2, se puede establecer el módulo de elasticidad recomendado para el material. Una vez obtenidos el módulo de elasticidad de la matriz y el porcentaje de boleos presentes en el conglomerado (determinado previamente) es posible aproximar un modulo de elasticidad para el conjunto. Se puede usar para esto el grafico 3 o la ecuación usada para su elaboración. Aunque los resultados hallados en este trabajo necesitan una validación rigurosa, se puede decir que los valores obtenidos varían en un rango bastante plausible y además son parecidos a ensayos físicos realizados en materiales similares, tal es el caso de los resultados de estudios en México, explicados anteriormente. Por ello, se considera que este trabajo constituye un buen inicio en el necesario e importante estudio del conglomerado de la ciudad de Cuenca. 


\section{BIBLIOGRAFÍA}

Alemán Velásquez, J.D., A. Pantoja Sánchez, S. Villegas Lesso, 2009. Estudios y diseño geotécnico de la cortina del P.H. La Yesca. Revista de la Sociedad Mexicana de Mecánica de Suelos, 15-24.

Erazo, M., 2009. Reportes internos. Facultad de Ingeniería. Universidad de Cuenca.

Geuzaine, C., J. Remacle, 2008. Gmsh Reference Manual, para Gmsh 2.2. http://www.geuz.org/gmsh/.

Holzapfel, G., 2006. Nonlinear Solid Mechanics, A Continuum Approach for Engineering. John Wiley and Sons, Ltd.

Kanit, T., S. Forest, I. Galliet, V. Mounoury, D. Jeulin, 2003. Determination of the size of the representative volume element for random composites: statistical and numerical approach. Int. J. Solids Struct., 40 (13-14), 3647-3679.

Nemat-Nasser, S., M. Hori, 1999. Micromechanics: Overall properties of heterogeneous solids (2nd Ed.). Elsevier Science Publishers, 810 págs.

Nuñez, A., J. Orellana, 2009. Optimización de la segmentación de imágenes orientada a la homogeneización de conglomerados. Tesis previa a la obtención del título de ingeniero, Universidad de Cuenca, Cuenca, Ecuador.

Ostoja-Starzewski, M., 2006. Material spatial randomness: From statistical to representative volume element. Probabilist. Eng. Mech., 21, 112-132.

Samaniego, E., L. Matute, C. Samaniego, K. Santacruz, J. Merchán, J. Orellana, P. Quinde, 2008. Informe Final del Proyecto Estudio Numérico y Experimental de un Elemento de Volumen Representativo de Conglomerado, Universidad de Cuenca, Cuenca, Ecuador.

Santacruz, K., 2009. Determinación de las Propiedades Elásticas del Conglomerado de las Formaciones Turi y Terrazas Fluvioglaciáricas de la Ciudad de Cuenca a partir de las Propiedades de su Matriz. Tesis previa a la obtención del título de magíster, Universidad de Cuenca, Cuenca, Ecuador. 